\title{
ALONGAMENTO INTERMITENTE E CONTÍNUO AUMENTAM A AMPLITUDE DE MOVIMENTO E REDUZEM A FORÇA DOS FLEXORES DE PUNHO
}

\author{
ESTIRAMIENTO INTERMITENTE Y CONTINUO AUMENTAN LA AMPLITUD DEL \\ MOVIMIENTO Y REDUCEN LA FUERZA DE LOS FLEXORES DE MUÑECA
}

Paulo Henrique Marchetti ${ }^{1,2}$

(Educador Físico)

Viviane de Jesus P. Mattos'

(Estudante de Educação Física)

Érica Paes Serpa' (Educadora Física)

Josinaldo Jarbas da Silva ${ }^{1}$

(Educador Físico)

Enrico Gori Soares ${ }^{1}$ (Educador Físico)

Antonio Claudio Paulodeto'

(Educador Físico)

Daniel Alves Correa'

(Educador Físico)

Guanis de Barros Vilela Junior

(Educador Físico)

Willy Andrade Gomes 1,3

(Educador Físico)

1. Faculdade de Ciências da Saúde (FACIS), UNIMEP, Piracicaba, SP, Brasil. 2. Universidade de São Paulo, Faculdade de Medicina, Instituto de Ortopedia e Traumatologia,

São Paulo, SP, Brasil.

3. Universidade Nove de Julho,

São Paulo, SP, Brasil.

\section{Correspondência:}

Universidade Metodista de

Piracicaba, Faculdade de Ciências da

Saúde, Programa de Pós-Graduação

em Ciências do Movimento

Humano, Grupo de Pesquisa em

Performance Humana.

Rodovia do Açúcar Km 156, Bloco 7,

Sala 42, Taquaral. Piracicaba,

SP. Brasil. 13423-070.

dr.pmarchetti@gmail.com

\section{RESUMO}

Introdução: A manipulação do intervalo entre séries pode influenciar o desempenho de atividades subsequentes. Objetivo: Comparar o efeito do intervalo de alongamento estático de forma continua e intermitente no desempenho de flexores de punho. Métodos: A amostra foi composta por 14 adultos jovens, hígidos, do gênero masculino (idade $31 \pm 9$ anos; estatura $178 \pm 0,7 \mathrm{~cm}$; massa $85 \pm 12 \mathrm{Kg}$ ). Foi avaliada a amplitude de movimento passivo (ADMP) em extensão de punho, a força isométrica máxima de flexão de punho associado a eletromiografia superficial antes e depois de dois protocolos de alongamento com diferentes intervalos entre séries. Para cada sujeito, um dos membros superiores foi alongado com o protocolo contínuo (CON) e o outro com o intermitente (INT), de forma aleatória. O protocolo CON consistiu na realização do alongamento durante 6 minutos ininterruptos, e o INT consistiu na realização de seis séries de 1 minuto por 20 segundos de intervalo entre as séries. A intensidade foi mantida a 70-90\% da percepção subjetiva de desconforto. Resultados: Os resultados de ADMP mostraram aumento significante entre as condições pré e pós-intervenção, em ambos os protocolos INT $\left(81^{\circ} \pm 10\right.$ e $\left.94^{\circ} \pm 10, P<0,001\right)$ e CON $\left(87^{\circ} \pm 12\right.$ e $\left.96^{\circ} \pm 11, P=0,004\right)$. Os resultados para o pico de

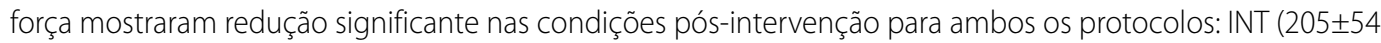
Kgf e $148 \pm 56$ Kgf, $P<0,001)$ e CON $(211 \pm 39$ Kgf e $144 \pm 36$ Kgf, $P<0,001)$. Os resultados para a taxa de produção de força mostraram aumento significante nas condições pré e pós-intervenção, para ambos os protocolos INT $(0,52 \pm 0,29 \mathrm{Kgf} / \mathrm{ms}$ e $1,24 \pm 0,45 \mathrm{Kgf} / \mathrm{ms}, P<0,001)$ e CON $(0,43 \pm 0,29 \mathrm{Kgf} / \mathrm{ms}$ e $1,11 \pm 0,34 \mathrm{Kgf} / \mathrm{ms}, P<0,001)$. Conclusão: Ambos os protocolos aumentaram a amplitude passiva de movimento, reduziram a força pico e taxa de produção de força, sem modificações na ativação dos flexores de punho.

Palavras-chave: exercício, treinamento de resistência, desempenho atlético.

\section{ABSTRACT}

Introduction: The management of rest interval among series can influence the performance of subsequent activities. Objective: To compare the effect of rest intervals during continuous and intermittent static stretching upon wrist flexor performance. Methods: The sample comprised 14 young, healthy male adults, (age: $31 \pm 9$ years, height: $178 \pm 0.7 \mathrm{~cm}$, weight: $85 \pm 12 \mathrm{~kg}$ ). Evaluation was carried out of the passive range of motion (PROM) in wrist extension, the maximum isometric strength of wrist flexion associated with surface electromyography before and after two stretching protocols with different intervals among series. For each subject, one of the upper limbs was stretched with the continuous protocol (CON) and the other with the intermittent (INT) protocol, randomly. As regards CON, it consisted in stretching during six uninterrupted minutes, whereas INT consisted in stretching six series of 1 minute for 20 seconds of rest interval among series. The intensity was kept at 70-90\% of the subjective perception of discomfort. Results: The results of PROM showed significant increase between pre and post-intervention conditions, in both protocols INT $\left(81^{\circ} \pm 10\right.$ and $\left.94^{\circ} \pm 10, P<0.001\right)$ and $\operatorname{CON}\left(87^{\circ} \pm 12\right.$ and $\left.96^{\circ} \pm 11, P=0.004\right)$. The results for peak force showed significant reduction in post-intervention conditions for both protocols: INT ( $205 \pm 54 \mathrm{Kgf}$ and $148 \pm 56 \mathrm{Kgf}, P<0.001)$ and $\mathrm{CON}(211 \pm 39 \mathrm{Kg}$ and $144 \pm 36 \mathrm{Kgf}, P<0.001)$. The results for the rate of force development showed significant increase in pre and post-intervention conditions for both protocols INT $(0.52 \pm 0.29 \mathrm{Kgf} / \mathrm{ms}$ and $1.24 \pm 0.45 \mathrm{Kgf} / \mathrm{ms}, P<0.001)$ and $\mathrm{CON}(0.43 \pm 0.29 \mathrm{Kgf} / \mathrm{ms}$ and $1.11 \pm 0.34$ $\mathrm{Kgf} / \mathrm{ms}, P<0.001)$. Conclusion: Both protocols increased the passive range of motion, reduced the peak force and the rate of force development, without changes in the activation of wrist flexors.

Keywords: exercise, resistance training, athletic performance.

\section{RESUMEN}

Introducción: El manejo de la pausa entre series puede influir en el desempeño de actividades posteriores. Objetivo: Comparar el efecto del intervalo de estiramiento estático de forma continua e intermitente en el desempeño de los flexores de la muñeca. Métodos: La muestra era composta por 14 jóvenes, varones y sanos (edad $31 \pm 9$ anos, altura $178 \pm 0,7 \mathrm{~cm}$, peso $85 \pm 12 \mathrm{~kg}$ ). Se evaluó la amplitud del movimiento pasivo (ADMP) en extensión de la muñeca, la fuerza máxima 
isométrica de la flexión de la muñeca asociada con electromiografía de superficie antes y después de dos protocolos de estiramientos con diferentes intervalos entre series. Para cada individuo, uno de los miembros superiores se alargó con el protocolo continuo (CON) y el otro con el intermitente (INT), al azar. El protocolo CON consistió en la realización de 6 minutos sin interrupciones durante el estiramiento, y el INT consistió en 6 series de 1 minuto por 20 segundos de intervalo entre series. La intensidad se mantuvo en 70-90\% de la percepción subjetiva de malestar. Resultados: Los resultados de ADMP mostraron aumento significativo entre las condiciones antes y después de la intervención, en ambos protocolos INT $\left(81^{\circ} \pm 10\right.$ y $\left.94^{\circ} \pm 10, P<0,001\right)$ y CON $\left(87^{\circ} \pm 12\right.$ y $\left.96^{\circ} \pm 11, P=0,004\right)$. Los resultados para el pico de fuerza mostraron reducción significativa en las condiciones posteriores a la intervención de ambos protocolos: INT (205 \pm 54 Kgf y $148 \pm 56 \mathrm{Kgf}, P<0,001)$ y CON (211 $\pm 39 \mathrm{Kg}$ y $144 \pm 36 \mathrm{Kgf}, P<0,001)$. Los resultados de la tasa de producción de fuerza mostraron aumento significativo en las condiciones pre- $y$ después de la intervención, para ambos protocolos

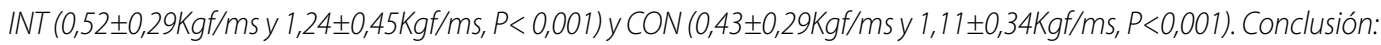
Ambos protocolos aumentaron la amplitud del movimiento pasivo, reducirán la fuerza máxima y la tasa de producción de fuerza, sin cambios en la activación de los flexores de la muñeca.

Palabras clave: ejercicio, entrenamiento de resistencia, rendimiento atlético.

\section{INTRODUÇÃO}

Diversos esportes e atividades recreativas utilizam os membros superiores no suporte ou transferência de força, e associam tais atividades à prática do alongamento como aquecimento ou mesmo como parte do processo de treinamento, visando o aumento da amplitude de movimento (ADM) (ex: judô, jiu-jitsu, basebol, ginástica olímpica, entre outros) ${ }^{1-5}$.

Entretanto, dependendo da forma como o alongamento é empregado no treinamento, este pode afetar diferentes sistemas biológicos como o neurofisiológico, hormonal, celular e mecânico, e influenciar de forma positiva ou negativa a resposta do tecido a estímulos subsequentes como a produção de força, potência ou o padrão de ativação muscular ${ }^{4-9}$. Diversos estudos demonstram efeitos deletérios na produção de força após exercícios de alongamento estático $5,6,10-13$ e redução da atividade muscular após os exercícios de alongamento ${ }^{8,14}$, os quais podem estar relacionados à ajustes em diferentes mecanismos como os de inibição neural e/ou de alteração tecidual na unidade músculo-tendínea5,6,15-17.

A manipulação das variáveis agudas do treino de flexibilidade, como o método (estático ou dinâmico), a intensidade, os intervalos entre séries, e a duração do alongamento podem influenciar o desempenho de atividades subsequentes ${ }^{5,18}$. De acordo com Freitas et al. ${ }^{18}$ a intensidade imposta durante o alongamento é um dos fatores determinantes para o aumento da amplitude de movimento (ADM) ou mesmo a redução do desempenho. Em estudo de Behm e Chaouachi ${ }^{5}$ verificaram que durações de alongamento acima de 270 segundos são mais eficientes quando o objetivo é aumentar a ADM.

Poucos são os estudos que avaliaram os efeitos dos intervalos entre séries de alongamento no desempenho ${ }^{19,20}$, que podem ser classificados como intermitentes ou contínuos. A maioria dos estudos que investigam a perda de força/potência utilizam protocolos chamados de intermitentes (ou seja, com intervalos de descanso), que podem levar à alterações negativas nas propriedades musculares (aspectos mecânicos) e neurais. O alongamento intermitente tem sido reportado como sendo mais eficiente na redução da rigidez muscular, quando comparado ao alongamento contínuo (sem intervalos de descanso), e que pode estar associado com a redução na viscosidade do músculo, no comportamento tixotrópico (histerese) do sistema músculo-articular, e alterações na cinética da oxigenação tecidual ${ }^{17,20}$. Trajano et al. ${ }^{20}$ analisaram as contribuições relativas dos fatores centrais e periféricos na queda de força induzida pelos protocolos de alongamento agudo de forma contínua (1×5 min) e intermitente ( $5 \times 1$ min) nos flexores plantares, avaliados através de um dinamômetro isocinético. Os resultados sugerem melhora na ADM de tornozelo para ambos os protocolos, entretanto o protocolo intermitente apresentou maior redução no pico de torque quando comparado ao protocolo contínuo. Outro estudo de Marchetti et al. ${ }^{19}$ comparou diferentes protocolos de alongamento estático com intervalos contínuos e intermitentes no desempenho do bounce drop jump, utilizando o mesmo volume e intensidade. O protocolo contínuo utilizou uma série de 6 minutos, e o intermitente seis séries de 1 minuto com 20 segundo entre estímulos. Ambos utilizaram uma intensidade alta de 70-90\% acima do ponto de desconforto. Os resultados do estudo mostraram redução no desempenho (altura de salto) e aumento da ADM em ambos os protocolos, entretanto o tempo de contato durante o salto aumentou apenas para o protocolo contínuo, mostrando uma provável maior redução na capacidade elástica do tecido quando o mesmo não recebe intervalos de recuperação (ou retorno ao comprimento inicial). Os resultados mostraram redução na altura de salto e ambos os protocolos (contínuo e intermitente), e aumento no tempo de contato somente para o protocolo contínuo. Assim, o objetivo do presente estudo foi comparar o efeito agudo do intervalo de alongamento estático utilizando a forma continua e intermitente no desempenho de flexores de punho.

\section{MÉTODOS}

O tamanho da amostra foi determinado a partir de um estudo piloto previamente realizado, utilizando indivíduos que possuíam as mesmas características daquelas que foram empregadas no presente estudo, baseando-se em uma significância de 5\% e um poder do teste de $80 \%{ }^{21}$. A amostra foi composta por 14 adultos jovens, hígidos, do gênero masculino (idade: $31 \pm 9$ anos, estatura: $178 \pm 0,7 \mathrm{~cm}$, massa corporal total: $85 \pm 12 \mathrm{Kg}$ ), sem quaisquer lesões e/ou desordens neurológicas periféricas e/ou centrais que pudessem acometer o desempenho de membros superiores, em especial a região do antebraço e articulação de punho. Os sujeitos foram informados dos procedimentos experimentais e assinaram o Termo de Consentimento Livre e Esclarecido, aprovado pelo Comitê de Ética da Universidade (№ 12/13).

\section{Procedimentos}

Os sujeitos foram informados para se abster de qualquer atividade física que envolvesse a área muscular envolvida nas últimas 48 horas. Inicialmente foram coletados os dados antropométricos (estatura, massa) e dados pessoais (nome, idade, e possíveis lesões ou desordens neurológicas), seguido de um breve aquecimento utilizando flexões de punho isométricas submáximas e familiarização com o teste de flexão isométrica de punho, realizada em ambos os antebraços. Então, 
foi avaliada a amplitude de movimento passivo (ADMP) em extensão de punho antes e depois dos protocolos de alongamento. Para cada sujeito, um dos membros superiores foi alongado com o protocolo contínuo e o outro com o intermitente, de forma aleatorizada entre sujeitos. Após os protocolos de alongamento, os sujeitos foram submetidos à avaliação isométrica da força máxima que foi integrada à atividade mioelétrica de flexores de punho.

Amplitude de movimento passivo (ADMP): A ADMP foi avaliada antes de depois de cada protocolo de alongamento passivo através de um flexímetro analógico (Sanny ${ }^{\circledR}$, Brasil). Para a avaliação da ADMP, os sujeitos permaneceram sentados, com um dos antebraços em supinação e apoiado em uma superfície plana e rígida. Em seguida, o flexímetro foi fixado lateralmente à palma da mão, de forma que o zero do marcador analógico ficasse posicionado perpendicularmente a superfície de apoio. Então, foi realizada uma flexão de punho passiva pelo pesquisador, até que os sujeitos atingissem sua ADMP máxima, para ambos os membros superiores separadamente. Todas as avaliações foram realizadas pelo mesmo pesquisador.

Eletromiografia Superficial (sEMG): Para a coleta dos dados de SEMG, foi utilizado um eletromiógrafo de 2 canais (EMG832C, EMG system Brasil, São José dos Campos, Brasil) com frequência de amostragem de $2000 \mathrm{~Hz}$, através de um software de aquisição (DATAQ Instruments Hardware Manager, DATAQ Instruments, Inc., OH, USA). A atividade sEMG foi amplificada (amplificador bi-polar diferencial, com impedância de entrada $=2 \mathrm{M} \Omega$, e taxa de rejeição de modo comum $>100 \mathrm{~dB}(60 \mathrm{~Hz})$, ganho $\times 100$, ruído $>5 \mu \mathrm{V}$ ), e um conversor AD de 12 bit. Para a colocação dos eletrodos os pelos foram removidos da região e uma leve abrasão foi realizada na pele para remoção das células mortas e redução da impedância. Foram utilizados pares de eletrodos ativos de superfície, circulares, autoadesivos, $\mathrm{Ag} / \mathrm{AgCl}$ com $1 \mathrm{~cm}$ de diâmetro, com espaçamento de $2 \mathrm{~cm}$ de centro a centro entre os eletrodos, associados à um gel condutor, sendo colocados sobre o músculo flexor radial do carpo, posicionados na linha entre o punho e o cotovelo a 1/3 de distância do cotovelo. A localização específica de cada eletrodo foi norteada segundo as recomendações de Cram et al..22. O eletrodo monopolar de referência autoadesivo, $\mathrm{Ag} / \mathrm{AgCl}$ com $2 \mathrm{~cm}$ de diâmetro, associado à um gel condutor, foi colocado na proeminência óssea da clavícula.

Teste de força isométrica máxima (FIM): O teste de flexão isométrica máxima de punho foi realizado através de um dinamômetro manual (CEFISE ${ }^{\circledR}$, Brasil) à uma frequência de aquisição do $100 \mathrm{~Hz}$. Os sujeitos permaneceram sentados, segurando o dinamômetro com a mão em posição supina, estando o antebraço e a mão totalmente apoiados sob uma superfície plana e rígida. A distância entre a célula de carga e a mão do sujeito foi ajustada para que a tarefa fosse executada de forma isométrica, com a articulação do punho na posição anatômica e a célula de carga foi fixada perpendicularmente ao antebraço/punho. O teste de FIMP consistiu na realização de duas tentativas máximas durante 10 seg com 1 min de intervalo entre tentativas.

Protocolo de Alongamento: Foram realizados dois protocolos distintos de alongamento estático passivo, (intermitente e contínuo) sendo um executado no braço direito e um no braço esquerdo de cada sujeito de forma aleatorizada e com 5 min de intervalo entre protocolos. O protocolo contínuo (CON) consistiu na realização do alongamento durante 6 minutos ininterruptos. Já o protocolo intermitente (INT) consistiu na realização do alongamento dividido em seis séries de $1 \mathrm{~min}$ por 20 seg de intervalo entre as séries. 0 volume total de ambos os protocolos foi equalizado, consistindo em 360 seg (músculo sob tensão), sendo os mesmos realizados sempre pelo mesmo pesquisador. Para garantir a homogeneidade em relação à intensidade do alongamento, foi utilizada uma escala subjetiva de desconforto (PSD), onde $0=$ "sem desconforto de alongamento" e 100\%="o máximo desconforto de alongamento imaginável"5,7,23. A intensidade do PSD foi mantida a 70-90\% do PSD e reajustada durante ambos os protocolos, se necessário.

\section{Análise de Dados}

Os dados de sEMG foram tratados para posterior comparação e análise conforme preconizado por Winter ${ }^{24}$, através de uma rotina escrita no software Matlab (Mathworks Inc., EUA). Foi removido o primeiro segundo do sinal EMG visando evitar ajustes corporais ou o efeito da fadiga e então utilizado para análise apenas três primeiros segundos de contração isométrica. O processamento do sinal sEMG seguiu a seguinte ordem: os sinais sEMG foram filtrados com um filtro de $4^{\mathrm{a}}$ ordem, passa banda entre $20-400 \mathrm{~Hz}$, e atraso de fase zero. Foi utilizada a root-mean square (RMS) com uma janela de 150 ms, para a amplitude do sinal EMG (RMS EMG). A atividade muscular foi então integrada (IEMG). Para a análise no domínio de frequências foi utilizada a transformada rápida de Fourier. A frequência mediana (FMed) do espectro de potência foi calculada para cada condição e utilizada para posterior análise. Os valores da IEMG e da FMed foram comparados nas condições de pré e pós-alongamento. Os dados de flexibilidade foram utilizados e comparados para verificar se houveram diferenças na ADMP entre as condições (pré e pós-alongamento) para ambos os protocolos.

Os dados da FIM em cada tentativa foram filtrados por um filtro Butterworth de quarta ordem, passa-baixa de $10 \mathrm{~Hz}$ e atraso de fase zero e então o maior valor das tentativas foi considerado. Os dados de força adquiridos através do dinamômetro manual foram analisados através das seguintes variáveis: (1) pico de força (maior valor da curva força $x$ tempo); (2) taxa de produção de força, calculado através da inclinação da reta da curva força $x$ tempo nos primeiros $150 \mathrm{~ms}$.

\section{Análise Estatística}

A normalidade e homogeneidade das variâncias foram verificadas utilizando o teste de Shapiro-Wilk e de Levene, respectivamente. Uma ANOVA (2x2) foi utilizada para verificar as diferenças entre as condições (pré e pós-alongamento) e protocolos (INT e CON) para as variáveis dependentes: IEMG, FMed, Pico de força, e TDF. A confiabilidade da variável dependente pico de força foi calculada e determinada utilizando o coeficiente de correlação intra-classe (CCI). Foram utilizados os seguintes critérios para o $\mathrm{CCl}$ : $<0,4$ pobre; 0,4 - < 0,75 satisfatório; $\geq 0,75$ excelente $^{25}$. O cálculo do efeito do tamanho (Effect Size, ES) foi realizado através da formula de Cohen e os resultados se basearam nos seguintes critérios: $<0,35$ efeito trivial; 0,35-0,80 pequeno efeito; 0,80-1,50 efeito moderado; e > 1,5 grande efeito, para sujeitos treinados de forma recreacional baseando em Rhea $^{26}$. Uma significância (a) de 5\% foi utilizada para todos os testes estatísticos, através do software SPSS versão 18.0.

\section{RESULTADOS}

Os resultados de ADMP mostraram aumento significante entre as condições pré e pós-intervenção, em ambos os protocolos INT $\left(81^{\circ} \pm 10\right.$ e $94^{\circ} \pm 10$, respectivamente, $\left.P<0,001 ; \mathrm{TE}=1,2 ; \Delta \%=14 \%\right)$ e CON $\left(87^{\circ} \pm 12\right.$ e $96^{\circ} \pm 11$, respectivamente, $\left.P=0,004 ; \mathrm{TE}=0,70 ; \Delta \%=9,3\right)$.

Para as variáveis IEMG (figura 1A) e FMed (figura 1B) não foram verificadas diferenças significantes entre protocolos (INT x CON) ou condições pré e pós-intervenção.

Os resultados para o pico de força mostraram redução significante nas condições pós-intervenção para em ambos os protocolos: INT $(205 \pm 54 \mathrm{Kgf}$ e $148 \pm 56 \mathrm{Kgf}$, respectivamente, $P<0,001$, TE=1,03; $\Delta \%=27,8 \%)$ e CON $(211 \pm 39 \mathrm{Kgf}$ e $144 \pm 36 \mathrm{Kgf}$, respectivamente, $P<0,001, \mathrm{TE}=1,78 ; \Delta \%=31,7 \%$ ) (figura $2 \mathrm{~A}$ ). 
Os resultados para a taxa de produção de força mostraram aumento significante nas condições pré e pós-intervenção, para ambos os protocolos INT $(0,52 \pm 0,29 \mathrm{Kgf} / \mathrm{ms}$ e 1,24 $\pm 0,45 \mathrm{Kgf} / \mathrm{ms}$, respectivamente, $P<0,001, \mathrm{TE}=1,9 ; \Delta \%=58,06 \%)$ e CON $(0,43 \pm 0,29 \mathrm{Kgf} / \mathrm{ms}$ e $1,11 \pm 0,34$ $\mathrm{Kgf} / \mathrm{ms}$, respectivamente, $P<0,001, \mathrm{TE}=2,15 ; \Delta \%=61,26 \%$ ) (figura $2 \mathrm{~B}$ ).

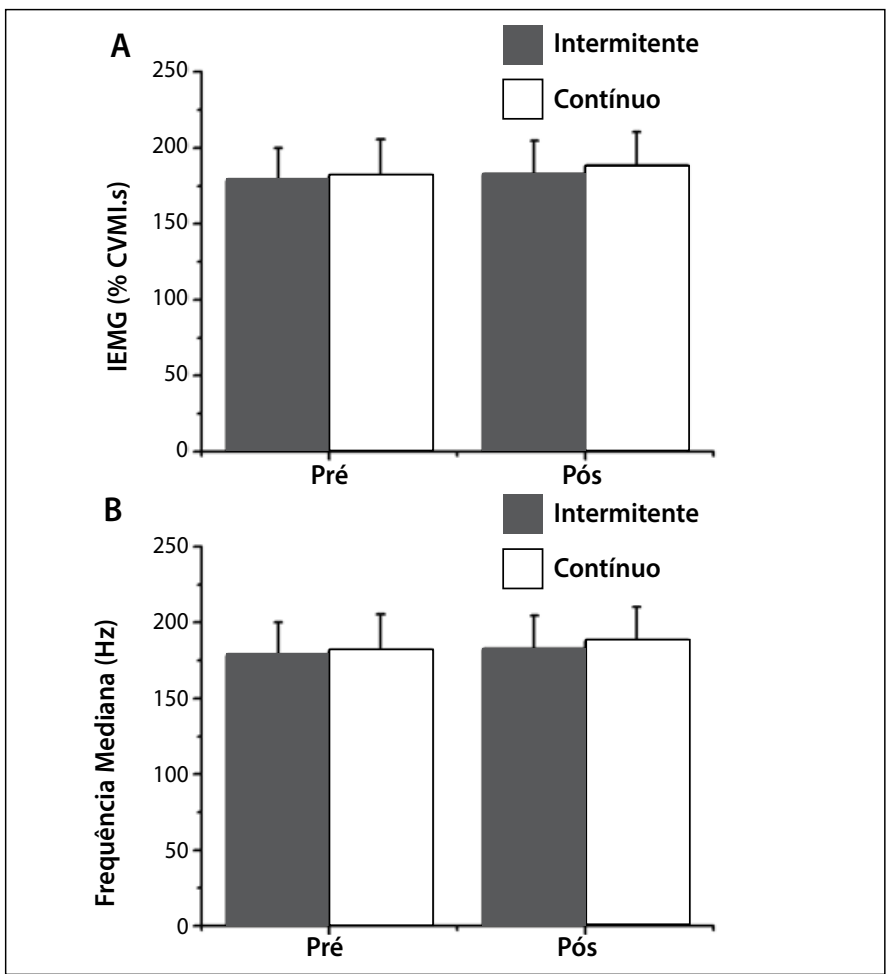

Figura 1. Média e desvio padrão da (A) IEMG e (B) frequência mediana durante a flexão isométrica máxima de punho, entre as condições pré e pós-intervenção (intermitente ou contínuo). $\left({ }^{*} \mathrm{P}<0,05\right)$.

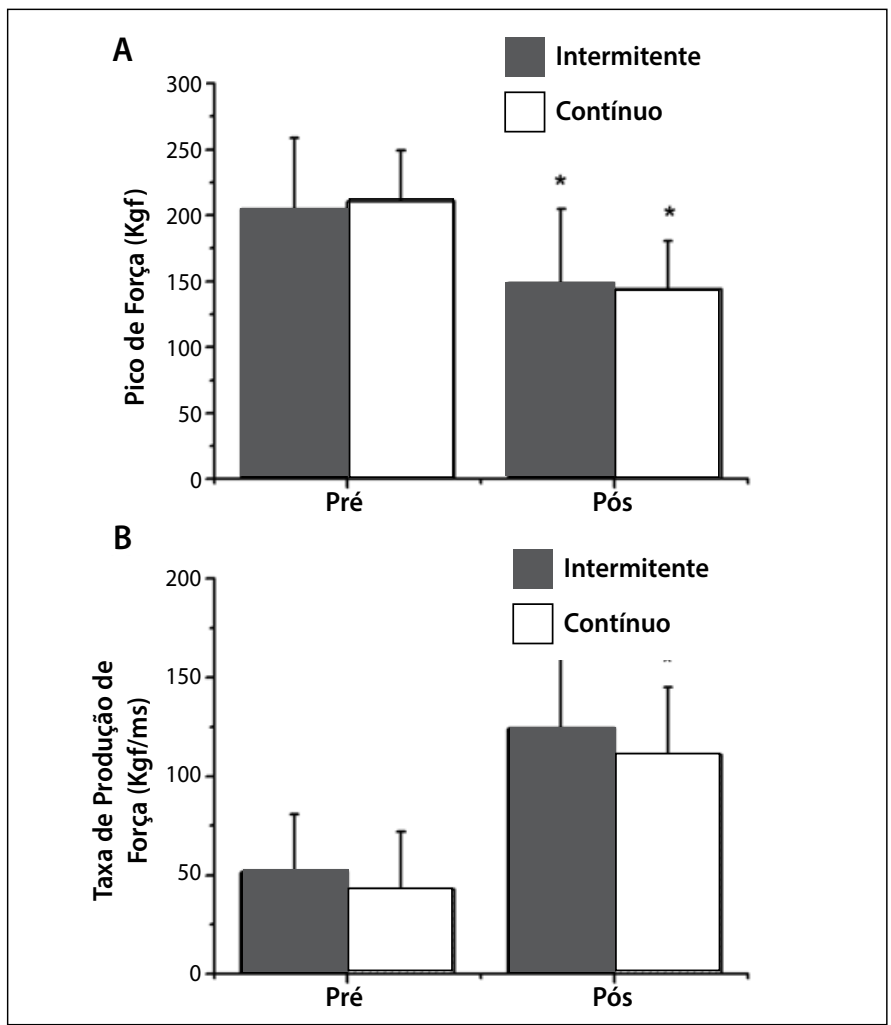

Figura 2. Média e desvio padrão do (a) pico de força nos primeiros 150ms da contração voluntária máxima e (b) taxa de produção de força durante a flexão isométrica máxima de punho, entre as condições pré e pós-intervenção (intermitente ou contínuo). ${ }^{*}<<0,05$

\section{DISCUSSÃO}

O presente estudo verificou os efeitos agudos do alongamento estático intermitente e continuo no desempenho máximo da flexão de punho, sendo que os principais resultados mostraram aumento na ADM, um aumento na taxa de produção de força e redução na força isométrica em ambos os protocolos.

Ambos os protocolos foram aplicados com a mesma duração total de 360 seg, e independente da duração total, ambos os protocolos foram eficientes para aumentar a amplitude de movimento passiva (ADMP), quando comparados ao momento pré-alongamento, entretanto, sem diferenças entre eles. A intensidade imposta, como parte da carga de treino, foi similar em ambos os protocolos, mesmo considerado como um dos fatores determinantes para o aumento da ADMP18, esta não parece ter sido a causa principal das alterações observadas no presente estudo. O aumento da ADMP pode ser atribuído a fatores mecânicos (redução da rigidez e redução na viscosidade), neurais ou a tolerância ao alongamento $5,7,15,27-31$.

Considerando que o alongamento passivo prolongado comumente apresenta reduções na excitabilidade dos motoneurônios avaliada através do sEMG ou da atividade do reflexo $\mathrm{H}^{32}$. Trajano et al. ${ }^{20} \mathrm{com}-$ pararam os efeitos de diferentes protocolos de alongamento e sua influencia sob o sistema nervoso central e periférico na produção de força. Participaram do estudo 18 jovens saudáveis sem limitações na rigidez articular e destreinados em alongamentos há pelo menos seis meses. Foram realizadas três condições experimentais: a) uma série com 5 min de alongamento contínuo (CON); b) Cinco séries de 1 min de alongamento intermitente (INT) com intervalo entre séries de 15 seg; c) uma condição controle (GC). Todas as condições experimentais foram aleatorizadas entre os sujeitos e realizadas em dias separados. Os protocolos de alongamento (CON e INT) foram realizados na articulação do tornozelo, visando alongar o músculo tríceps sural através de um dinamômetro isocinético a uma intensidade de 90\% do máximo tolerável por cada sujeito. Durante o alongamento foram avaliados: o nível de oxigenação do gastrocnêmio medial através de uma espectroscopia infravermelho, o pico de torque $\left(T_{\text {pico }}\right)$, a ativação voluntária (AV\%, técnica de interpolação do twich), a amplitude sEMG normalizada pela $M_{\max }$ (EMG: M), amplitude da onda $V$ excitação-contração (EC), a eficiência de acoplamento (relação do torque por estimulação tetânica entre 20 e $80 \mathrm{~Hz}$ [20:80]), nos momentos pré-, imediatamente após, 15 e 30 min após cada condição experimental. Os resultados mostraram que ambos os protocolos de alongamento (INT e CON) reduziram significativamente a oxigenação dos tecidos, no entanto, o alongamento CON resultou em maior queda nas concentrações de oxi-hemoglobina. OT pico avaliado imediatamente após o alongamento apresentou redução em ambos os protocolos, tendo maior redução em INT (-23,8\%) do que CON (-14,3\%). Para os momentos 15 e 30 min após o alongamento apenas o INT apresentou redução significante. Para a sEMG:M e AV\% foi verificada redução apenas para INT (-27,7\% e $-15,9 \%$ respectivamente). Além disso, foi verificada correlação entre a redução no $T_{\text {pico }}$ para ambos os protocolos de alongamento e a redução da EMG:H (CON: r=0,81 e INT: $r=0,89)$, AV\% (CON: $r=0,78$ e INT: $r=0,93)$ e onda $V(r=0,51$, somente para INT). Ambos os protocolos de alongamento reduziram o torque por estimulação tetânica de 20 e 80 Hz (INT: $-13,1 \%$ e -6,4\% e CON: -10,9\% e -6,7\%), mas não para o grupo controle. Concluindo que ambos os protocolos de alongamento reduziram o $T_{\text {pico, }}$ no entanto, o protocolo INT foi mais eficiente na redução do $T_{\text {pico }}$ do que o CON, estando este, fortemente associado a certa redução da capacidade do sistema nervoso central. O presente estudo não corrobora os achados de Trajano et al. ${ }^{20}$, pois não foram verificadas diferenças na ativação muscular dos flexores durante a tarefa de flexão 
de punho isométrico. Entretanto, no presente estudo as variáveis de desempenho analisadas apresentam informações adicionais para as condições de potência (alta velocidade de contração), como a força pico e a taxa de desenvolvimento de força (TDF), sendo que estas foram afetadas negativamente pelo alongamento, independe do tipo de intervalo realizado, e assim descartando quaisquer efeitos neurais aparentes. Entretanto, o alongamento passivo pode ter afetado a rigidez ou mesmo a transferência de forças na unidade músculo tendínea em função de alterações nas estruturas passivas, as quais transferem a tensão rapidamente para o movimento. Outra possiblidade seria uma alteração da histerese da unidade músculo tendínea, a qual pode ser caracterizada pela perda de energia (conversão em calor) nos tecidos viscoelásticos quando submetidos a cargas e descargas ${ }^{15,17}$.

\section{REFERÊNCIAS}

1. Alter MJ. Ciência da flexibilidade. 2a ed. São Paulo: Artmed; 1999

2. Marchetti PH, Soares EG, Silva FHDO, Medeiros II, Reis Neto I, Lopes CR, et al. Efeito de diferentes durações do alongamento no desempenho de saltos unipodais. Rev Bras Med Esporte. 2014;20(3):223-6.

3. Behm DG, Bambury A, Cahill F, Power K. Effect of acute static stretching on force, balance, reaction time, and movement time. Med Sci Sports Exerc. 2004;36(8):1397-402.

4. Behm DG, Button DC, Butt JC. Factors affecting force loss with prolonged stretching. Can J Appl Physiol. 2001;26(3):261-72.

5. Behm DG, Chaouachi A. A review of the acute effects of static and dynamic stretching on performance. Eur J Appl Physiol. 2011;111(11):2633-51.

6. Rubini EC, Costa AL, Gomes PS. The effects of stretching on strength performance. Sports Med. 2007;37(3):213-24.

7. Behm DG, Kibele A. Effects of differing intensities of static stretching on jump performance. Eur J Appl Physiol. 2007 Nov;101(5):587-94

8. Cornwell A, Nelson AG, Sidaway B. Acute effects of stretching on the neuromechanical properties of the triceps surae muscle complex. Eur J Appl Physiol. 2002;86(5):428-34.

9. Wallmann HW, Mercer JA, McWhorter JW. Surface electromyographic assessment of the effect of static stretching of the gastrocnemius on vertical jump performance. J Strength Cond Res. 2005;19(3):684-8.

10. Kay AD, Blazevich AJ. Effect of acute static stretch on maximal muscle performance: a systematic review. Med Sci Sports Exerc. 2012;44(1):154-64.

11. Herda TJ, Cramer JT, Ryan ED, McHugh MP, Stout JR. Acute effects of static versus dynamic stretching on isometric peak torque, electromyography, and mechanomyography of the biceps femoris muscle. J Strength Cond Res. 2008;22(3):809-17.

12. Cramer JT, Beck TW, Housh TJ, Massey LL, Marek SM, Danglemeier S, et al. Acute effects of static stretching on characteristics of the isokinetic angle - torque relationship, surface electromyography, and mechanomyography. J Sports Sci. 2007;25(6):687-98.

13. Peck E, Chomko G, Gaz DV, Farrell AM. The effects of stretching on performance. Curr Sports Med Rep. 2014;13(3):179-85.

14. Marek SM, Cramer JT, Fincher AL, Massey LL, Dangelmaier SM, Purkayastha S, Fitz KA, Culbertson JY. Acute Effects of Static and Proprioceptive Neuromuscular Facilitation Stretching on Muscle Strength and Power Output. J Athl Train. 2005;40(2):94-103.

15. Serpa EP, Vilela Junior GB, Marchetti PH. Aspectos biomêcanicos da unidade músculo-tendínea sob efeito do alongamento. Revista CPAQV. 2014;6(1):1-14

16. Knudson D. The Biomechanics of Stretching. JESP. 2006;2:3-12
O presente estudo apresenta limitações quanto ao número de sujeitos analisados e o possível crosstalk sob os eletrodos posicionados no antebraço dos sujeitos.

\section{CONCLUSÃO}

Conclui-se que uma sessão aguda de alongamento estático passivo aumenta a amplitude passiva de movimento, reduz a força pico e taxa de produção de força e não apresenta modificações na ativação muscular de flexores de punho, independente do tipo de intervalo utilizado.

Todos os autores declararam não haver qualquer potencial conflito de interesses referente a este artigo.
17. Ozkaya N, Nordin M. Fundamentals of biomechanics: equilibrium, motion, and Deformation. 2nd ed New York: Springer; 1999.

18. Freitas SR, Vilarinho D, Vaz JR, Bruno PM, Costa PB, Mil-Homens P. Responses to static stretching are dependent on stretch intensity and duration. Clin Physiol Funct Imaging. 2014 Aug 27. doi: 10.1111/ cpf.12186. [Epub ahead of print]

19. Marchetti PH, Soares EG, Silva FHDO, Nardi PSM, Serpa EP, Gomes WA, et al. Acute effects of stretching routines with and without rest intervals between sets in the bounce drop jump perfrmance. Int $J$ Sports Sci Coach. 2015;5(1):1-5.

20. Trajano GS, Nosaka K, B Seitz L, Blazevich AJ. Intermittent stretch reducesforce and central drive more than continuous stretch. Med Sci Sports Exerc. 2014;46(5):902-10.

21. Eng J. Sample size estimation: how many individuals should be studied? Radiology. 2003;227(2):309-13.

22. Cram JR, Kassman GS, Holtz J. Introduction to surface electromyography. Gaithersburg, Maryland: Aspen Publication; 1998.

23. Marchetti PH, Silva FH, Soares EG, Serpa EP, Nardi PS, Vilela Gde B, Behm DG. Upper limb static-stretching protocol decreases maximal concentric jump performance. J Sports Sci Med. 2014;13(4):945-50.30.

24. Winter DA. Biomechanics and motor control of human movement. 4th ed. USA: Wiley; 1990.

25. Rosner B. Fundamentals of Biostatistics. 7th ed. Boston, MA: Cengage Learning; 2010.

26. Rhea MR. Determining the magnitude of treatment effects in strength training research through the use of the effect size. J Strength Cond Res. 2004;18(4):918-20.

27. Magnusson SP, Simonsen EB, Dyhre-Poulsen P, Aagaard P, Mohr T, Kjaer M. Viscoelastic stress relaxation during static stretch in human skeletal muscle in the absence of EMG activity. Scand J Med Sci Sports. 1996;6(6):323-8.

28. Magnusson SP, Simonsen EB, Aagaard P, Sørensen H, Kjaer M. A mechanism for altered flexibility in human skeletal muscle. J Physiol. 1996;497(Pt 1):291-8.

29. Magnusson SP, Simonsen EB, Aagaard P, Kjaer M. Biomechanical responses to repeated stretches in human hamstring muscle in vivo. Am J Sports Med. 1996;24(5):622-8.

30. Magnusson SP, Simonsen EB, Aagaard P, Dyhre-Poulsen P, McHugh MP, Kjaer M. Mechanical and physical responses to stretching with and without preisometric contraction in human skeletal muscle. Arch Phys Med Rehabil. 1996;77(4):373-8.

31. Blazevich AJ, Cannavan D, Waugh CM, Fath F, Miller SC, Kay AD. Neuromuscular factors influencing the maximum stretch limit of the human plantar flexors. J Appl Physiol (1985). 2012;113(9):1446-55.

32. Behm D, Power K, Drinkwater E. Comparison of interpolation and central activation ratios as measures of muscle inactivation. Muscle Nerve. 2001;24(7):925-34. 